\title{
A Novel Technique to Detect Faces in a Group Photo
}

\author{
Saravanan Chandran \\ Assistant Professor, National Institute of Technology, Durgapur, West Bengal, India.
}

\begin{abstract}
Digital Image Processing plays a vital role in several applications. One of the applications is detection of people faces in a digital photo. Several research works have been progressing in the field of face identification and matching, facial expressions identification, smile detection, etc. Any application related to face, first step is detection of face. Thus, the face detection plays a key role. The face detection is a complicated process due to background objects, orientation, scale, lighting, geometrics, quality of image, occlusion, disguise etc. This paper discusses different research techniques published in the peer reviewed journal and proposed a technique to detect faces in a group photo.
\end{abstract}

\section{General Terms}

Image Processing.

\section{Keywords}

Face detection, object identification, biometrics.

\section{INTRODUCTION}

Digital image processing application plays a vibrant role in several applications which in turn makes our day to day activity simple and easy. Several research works are progressing in the area of image acquisition, image restoration, image compression, image representation, image fusion, object identification, etc. [1],[2],[12]-[17].

One of the important applications of image processing under object identification is Face Detection. The face detection is the first step in several applications such as surveillance and security, human computer interaction, object based video coding, virtual and augmented reality, automatic 3-D face modeling, gesture recognition, etc. [3]-[6].

In general the face detection applications are classified in to four categories, feature based, knowledge based, appearance based and template matching [3]. The features are detected based on the eyes, mouth, nose, eyebrow and cheek. The eye in the picture may have high intensity variance. The eyebrows, pupils and lips are darker and nose tip will be lighter in colour. The distance between mouth, left eye, right eye, and nose are used for detecting the face. Also the angle between the eyes, nose and the mouth are used to differentiate the face from other objects in the image [5].

\section{RECENT DEVELOPMENTS}

Several research papers have been published in the area of Face Detection. Selected research works will be discussed in this section for understanding the issues and techniques used for handling the issues.

H.H.K Tin has proposed robust approach for frontal face detection in colour images based on facial feature extraction and appearance based properties of face images [3]. He used the face detection algorithm proposed by Menser and Muller based on PCA. This approach extracts facial feature by segmenting the image regions using chrominance properties to detect facial features, such as eyes, nose and mouth, based on their statistical, structural, and geometrical relationships in frontal face images. The following figure 1 represents the procedure he adopted.

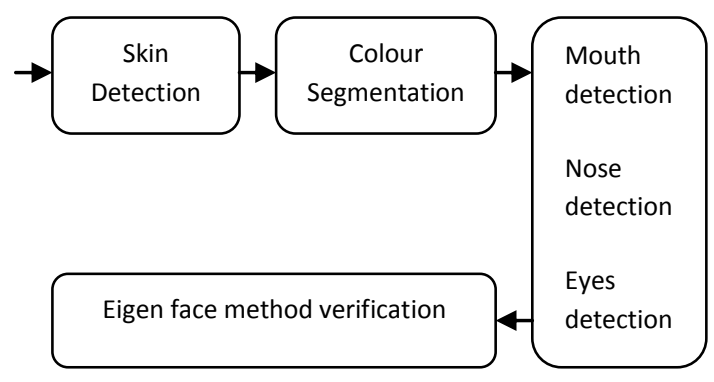

Fig 1: Procedure of Face Detection.

There were 400 face images were used as the set of training images taken from the internet. The images were a collection of 100 frontal upright images, 50 frontal images with glasses and 50 slightly rotated face images selectively chosen from the internet. From the results he observed that the spectacles of a person made reflection and lead to small amount of error.

H. Naeem [4] has proposed face detection system based on eigenfaces method. It had three major modules, preprocessing, construction of face space, and face localisation. A set of images were represented as long vector. This produced face space with each image representing a single point. The eigenfaces helped to detect lower dimensional space in which shorter vectors will describe face images. The following figure 2 represents face space shown in the article.

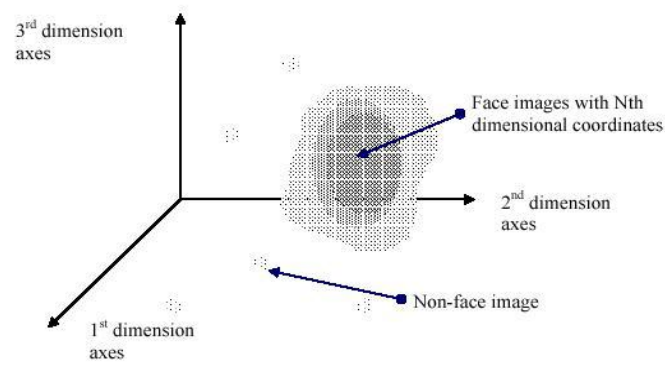

Fig 2: Face Space.

A. Mall and S. Ghosh [5] proposed neural network based face detection approach. They pre-processed input images using affine lighting correction. They applied neural network detectors $20 \times 20$ or $10 \times 10$ or $5 \times 5$ pixel region of an image then using an arbitrator the outputs are combined. They examined each location in the image in several scales and generated an output 1 or -1 . They used 1046 face images and 120 non-face images. They trained the network with $50 \%$ positive and $50 \%$ negative images. They improved the face 
detection rate.

O.Jesorsky, et.al, [6] proposed face detection using Hausdorff Distance. They used XM2VTS database and BIOID database and images were pre-processed to grayscale then made to $360 \times 288$ pixels and $384 \times 288$ pixels respectively. Their method had coarse detection and refinement phases each containing segmentation and localization step. They validated performance by relative error measure based on the distances between the expected and the estimated eye positions. They calculated average processing time per frame on a PIII $850 \mathrm{MHz}$ PC system is $23.5 \mathrm{~ms}$ for the coarse detection step and an additional $7.0 \mathrm{~ms}$ for the refinement step, which allowed the use in real time video applications. They show the experiment results XM2VTS obtained $98.4 \%$ and the BIOID obtained $91.8 \%$ accuracy.

M. Matsugu, et al, [7] proposed face detection using convolutional neural network and facial expression recognition. They have used $k$ modules to detect face. They used detection result of skin colour area as input to the face detection module 4. They obtained skin area simply by thresholding hue data of input image in the range of [20.078, 0.255] for the full range of [20.5, 0.5]. They have used standard back-propagation algorithm. They have used 22,890 face images and 137 non face images for testing of various sizes starting from $30 \times 30$ to $240 \times 240$. From the results it is observed that the $1 \%$ false rejection rate and $6 \%$ false acceptance rate.

K.T.Talele, et al., [8] proposed face detection using adaboost which combine simple statistical learners. AdaBoost selects a small set of features from available set of 180,000 features. They determined the three main parameters of cascade structure: The total number of classifiers $\mathrm{K}$, the number of features $n_{i}$ of each stage $i$, the threshold $\theta_{i}$ of each stage $i$. They tested the algorithm on one face image, two face image upto 10 face images of frontal face images.All faces in these images were perfectly detected but in case of group images the size of the face varies hence the algorithm sometimes failed in these cases.

P.S. Hiremath and M. Hiremath [9] proposed fuzzy face model using eyes and mouth features. In this proposed approach, the input image pre-processed and then the eyes are searched on the basis of geometrical knowledge of the symmetrical relations between eyes. They searched other prominent feature mouth using fuzzy rules and face detection algorithm with respect to the detected eyes. They used 700 test images from FERET, Biometric Ideal database, MIT, ORL, and CMU databases of type single frontal view face. These images were pre-processed to gray scale image. These gray scale imagesare filtered using the Sobel horizontal edge emphasizing filter. They observed in the filtered image, objects of interest (facial features) are brighter than the background. Further, they converted filtered image into binary image by simple global thresholding. Then, theydenoisedthe image by morphological operations, in which opening operation was performed to remove noise, and then the closing operation was performed to remove holes. They achieved $96.10 \%$ detection rate.

P.M.Balasubramaniam and A.Sathishkumar [10] proposed face detection using image fusion method. Their scheme had three stages, pre-processing, feature extraction, and learning based classification. They used skin segmentation, morphological opening, and region labelling in the first stage. They used DCT, DWT, Hu moment invariants, and the Canny edge detection for extracting the features. They used neural network to classify face or non-face in the last stage.

Devendra Singh Raghuvanshi and DheerajAgrawal [11] proposed face detection using skin colour segmentation, face features and regions properties. They used four colour models, $\mathrm{RGB}, \mathrm{YCbCr}, \mathrm{HSV}$ and CIELAB for segmenting the face. Then they converted the segmented image to binary image. Further, they used Euler number, bounding box properties, centroid, eccentricity like oval estimation, to identify the face. They used 30 images for testing with single face and multiple faces. From the experiment results they observed that the face detection percentage is $92 \%$.

From the literature survey it is observed generally, if the computation time increases, the face detection rate also increases; if the computation time decreases the face detection rate also decreases. Also, the size of the human face, background objects, lighting of the photograph, occlusion, disguise, are the key factors affecting the face identification process. It is decided to coin an algorithm to identify faces in a group photo of different background, light, scale, etc.

\section{PROPOSED TECHNIQUE}

A simple procedure made to evaluate face detection process in a set of group photos. Different group photos were collected using Google from various web sites. The group photos are different in size, different in texture, different in angle, different in lighting, different in background, and different number of faces in the photo at different points. A simple program developed in the MATLAB application for evaluate these photos.

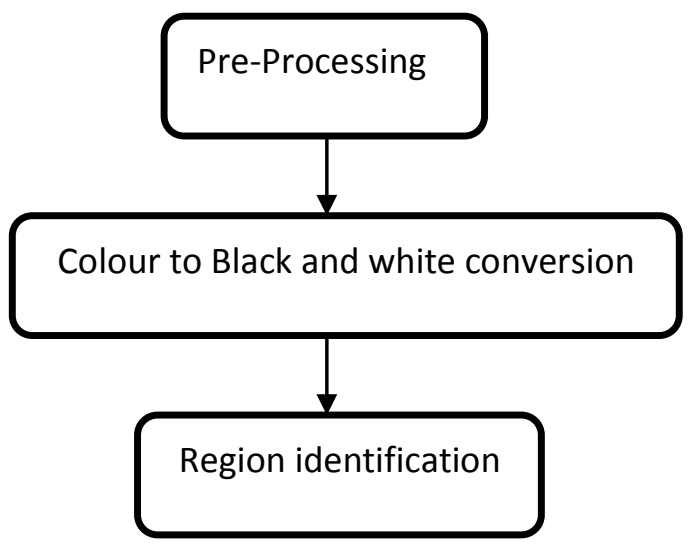

As a first step, shall be named as pre-processing step, a standard 2D average filter is applied on the picture to remove noises from the pictures. Second step, the colour image is converted to black and white. Third step, the boundaries are identified using standard region based boundary function. Finally the boundaries are marked with red based on the $\mathrm{k}$ and $\mathrm{N}$ values.

The experiment has been conducted to analyse the various factors affecting the face region identification process. The experiment shows the pre-processing filters affects the region identification. The average filter used with different horizontal size on several group photos then the regions are counted 
using boundary identification algorithm. With the smallest horizontal values the number of regions identified is higher and with higher horizontal values the number of regions identified is less. The following table 1 shows the value of ' $h$ ', regions identified, true face regions identified, false face regions identified, face regions not identified in a photo. From this table it is observed that the suitable value of a filter helps to identify the number of face regions accurately. From this table 1 , the value of $\mathrm{h}=8$, identifies more number of face regions, i.e., 17 , whereas value of $h=2$, identifies only 13 . The optimum value of ' $h$ ' is 10 for this picture. The optimum value of ' $h$ ' varies from picture to picture.

Table 1.Value of ' $h$ ' and regions values.

\begin{tabular}{|c|c|c|c|c|}
\hline $\begin{array}{c}\text { Valueof } \\
\text { 'h' }\end{array}$ & Regions & $\begin{array}{c}\text { True } \\
\text { face } \\
\text { regions }\end{array}$ & $\begin{array}{c}\text { False } \\
\text { face } \\
\text { regions }\end{array}$ & $\begin{array}{c}\text { Face regions } \\
\text { not } \\
\text { identified }\end{array}$ \\
\hline 2 & 47 & 13 & 2 & 11 \\
\hline 4 & 33 & 16 & 1 & 8 \\
\hline 6 & 24 & 16 & 2 & 8 \\
\hline 8 & 21 & 17 & 2 & 7 \\
\hline 10 & 19 & 17 & 2 & 7 \\
\hline 12 & 19 & 17 & 2 & 7 \\
\hline
\end{tabular}

Further, from the experiment it is noticed, the regions having large area and the regions having shorter area are not the face regions. In a group photo the regions of faces are having almost close region area values. Therefore, the face regions can be easily identified based on the closer region values. The large and small region values are ignored in our program. One of the source image used for experiment is shown below.

It is noticed from the image of the figure 4 , the face regions having opening in the face region are not identified as face region. The face regions having complete oval shape are identified successfully.

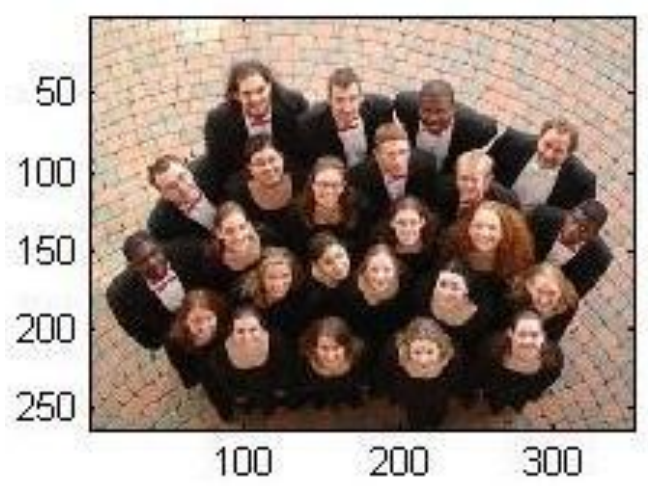

Fig 3: Source colour image

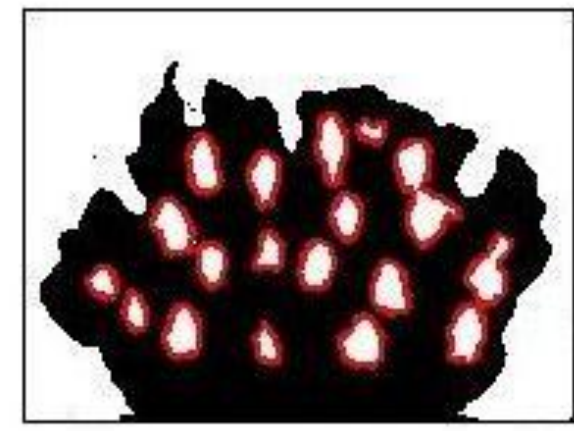

Fig 4: Face identified image with ' $h$ ' value 10

\section{CONCLUSION}

The proposed technique is simple and fast. The proposed algorithm works well for the group pictures having good lighting and without any occlusion and disguise in the face region. In the above picture the true face region identification ratio is $70.83 \%$, false face region identification ratio is $8.33 \%$ and the face regions not identified ratio is $29.17 \%$. In the future, the pre-processing step has to be improved to complete the open face regions. The open face regions have to be identified. Suitable algorithm shall be developed to close the open region. In the above picture, it is noticed two other regions matching the region area of the face regions are identified. In future, the region properties have to be matched with template of a face region having face properties in order to confirm the face region. This program is unable to identify face region for few group photos having same face region colour in other regions.

\section{REFERENCES}

[1] R. C. Gonzalez and R. E. Woods, Digital Image Processing, Prentice Hall.

[2] Gonzalez, R. C., R. E. Woods, and S. L. Eddins, Digital Image Processing Using MATLAB, New Jersey, Pearson Prentice Hall, 2004.

[3] HlaingHtakeKhaung Tin, Robust Algorithm for Face Detection in Color, I.J.Modern Education and Computer Science, 2012, 2, 31-37.

[4] HashaamNaeem, Face Detection using Eigen Method, International Journal of Engineering Science and Technology (IJEST), Vol. 4 No.03 March 2012 882-888.

[5] Anjana Mall, Mrs. ShusmitaGhosh, A Neural Network Based Face Detection Approach, Int.J.Computer Technology \&Applications, Vol 3 (2), 823-829.

[6] Oliver Jesorsky, et.al, Robust Face Detection Using the Hausdorff Distance, Lecture Notes in Computer Science, LNCS-2091, pp. 90-95, Halmstad, Sweden, 6-8 June 2001.

[7] Masakazu Matsugu, et al, Subject Independent Facial Expression Recognition with Robust Face Detection using a Convolutional Neural Network, Neural Networks 16 (2003) 555-559. 
[8] K.T.Talele, et al., Efficient Face Detection using Adaboost, International Journal of Computer Applications, Year:2012 Vol.:iccia Issue:10.

[9] Hiremath P.S. and ManjunathHiremath, Fuzzy Face Model for Face Detection using Eyes and Mouth Features, International Journal of Machine Intelligence, Volume 3, Issue 4, 2011, pp-185-190.

[10] P.M.Balasubramaniam and A.Sathishkumar, Detection of Human Faces using Image Fusion Method, International Journal of Engineering Science and Technology (IJEST), Vol. 3 No. 6 June 2011, 5408-5413.

[11] Devendra Singh Raghuvanshi and DheerajAgrawal, Human Face Detection by using Skin Color Segmentation, Face Features and Regions Properties, International Journal of Computer Applications (0975 8887) Volume 38-No.9, January 2012.

[12] C. Saravanan and R. Ponalagusamy "Fast Arithmetic Coding for Lossy Image Compression", International Journal of Computer, Mathematical Sciences and Applications, Vol. 1, No. 2-4, pp.257-265, 2007.

[13] C. Saravanan and R. Ponalagusamy "Medical Image Compression using Bi-Orthogonal Wavelets and Arithmetic Coding", International Journal of Computer,
Mathematical Sciences and Applications, Vol. 2 No. 2, pp.81-88, 2008.

[14] C. Saravanan and R. Ponalagusamy, "Lossless Greyscale Image Compression using Source Symbols Reduction and Huffman Coding", International Journal of Image Processing (CSC Journals), Vol.3, Iss.5, pp.246-251, 2009.

[15] C. Saravanan and R. Ponalagusamy "Gaussian noise estimation for Gray scale images using Mean value", Journal of Theoretical and Applied Information Technology, Vol.3, No.4, pp.68-73, 2007.

[16] C. Saravanan, Color Image to Grayscale Image Conversion, IEEE International Conference on Telecom Technology and Applications, Indonesia, March 19-21, 2010.

[17] C. Saravanan, Piyush Gupta, Pratima Sharma, Grey Scale Image Colorization Using Artificial Neural Network, WorldComp 2010 - The 2010 World Congress in Computer Science, Computer Engineering, and Applied Computing, Las Vegas, Nevada, USA, July 12-15, 2010 , pp.437-440. 